\title{
ASYMPTOTIC BEHAVIOR OF SOLUTIONS OF THE DAMPED BOUSSINESQ EQUATION IN TWO SPACE DIMENSIONS
}

\author{
VLADIMIR V. VARLAMOV
}

(Received 15 March 1997 and in revised form 25 April 1997)

\begin{abstract}
The Cauchy problem for the damped Boussinesq equation with small initial data is considered in two space dimensions. Existence and uniqueness of its classical solution is proved and the solution is constructed in the form of a series. The major term of its long-time asymptotics is calculated explicitly and a uniform in space estimate of the residual term is given.
\end{abstract}

Keywords and phrases. Boussinesq equation in two space dimensions, Cauchy problem, long-time asymptotics.

1991 Mathematics Subject Classification. 35Q20, 76B15.

1. Introduction. In recent years, the studies of nonlinear waves in media with dispersion have attracted attention of many mathematicians and physicists. One of the equations describing such processes is the Boussinesq one which was derived in the paper [6] and governs the propagation of long waves on the surface of shallow water. It can be written as

$$
u_{t t}=-\alpha u_{x x x x}+u_{x x}+\beta\left(u^{2}\right)_{x x}, \quad x \in \mathbb{R}^{1}, t>0,
$$

where $u(x, t)$ is an elevation of the free surface of fluid. Subscripts denote partial derivatives, and $\alpha, \beta=$ const $\in \mathbb{R}^{1}$ depend on the depth of fluid and the characteristic speed of propagation of long waves. Interesting comments on the derivation of (1.1) may be found in the survey [16]. It should be emphasized that the Boussinesq equation admits both right- and left-running wave solutions while the well-known Kortewegde-Vries one governs waves traveling in only one direction.

There is extensive literature on equation (1.1) (see, for example, $[8,9,12,13,15$, $14,19,28$ ], and the references there) which has been studied from various points of view. Hirota [12] has deduced conservation laws and has examined $N$-soliton interaction, and Clarkson [8] has given a general approach to construct exact solutions. Nonlinear evolution of a linearly stable solution has been investigated by Yajima [26]. Zakharov [28] has constructed the Lax pair for the inverse scattering transform. Nakamura [17] has discovered the explode-decay solitary wave solutions of the "spherical" Boussinesq equation, and Hirota [13] has applied the Wronskian technique for finding rational solutions of the spherical and the classical Boussinesq equations. The representation of periodic waves as sums of solitons has been given by Whitham [25]. Abstract Cauchy problems for the generalizations of (1.1) in Banach spaces have been 
considered in [14, 15, 19], where some sufficient conditions for the blow up of solutions in finite time have been presented.

Equation (1.1) takes into account dispersion, but in real processes, viscosity, also, plays an important role. Therefore, it is interesting to consider the equation

$$
u_{t t}-2 b u_{t x x}=-\alpha u_{x x x x}+u_{x x}+\beta\left(u^{2}\right)_{x x}, \quad x \in \mathbb{R}^{1}, t>0,
$$

which differs from the classical Boussinesq equation in the second term on the lefthand side accounting for dissipation. Here, $\alpha, b=$ const $>0, \beta=$ const $\in \mathbb{R}^{1}$.

In the papers $[5,3,4]$, Biler has examined some abstract Cauchy problems for the generalization of (1.2). Strictly speaking, the nonlinearity considered there was different (it contained some powers of the $L_{2}$-norm of the solution in question), and the "oscillation condition" used in [5] in the case of constant coefficients (1.2) took the form $\alpha>b^{2}$ (this condition is, also, used in the present paper). The author has obtained sufficient conditions for both power and exponential decay in time of some norms of the solution. In [27], You has considered (1.2) from a different point of view. He has applied the theory of global attractors and inertial manifolds to this equation. However, as far as the author of the present note knows, long time asymptotic expansions of solutions of the classical Cauchy problems for this equation have not yet been obtained.

The present paper is a continuation of the investigations [20, 22, 23, 21] on studying classical solutions of the Cauchy problems for equation (1.2) and it deals with the twodimensional case in space. It is well known that one of the methods of examining such problems for nonlinear evolution equations is the inverse scattering transform [1, 7]. Another approach has been proposed by Naumkin and Shishmarev [18], who have studied nonlocal nonlinear equations of the first order in time and with small initial data. They have used both the spectral and the perturbation theories for obtaining long time asymptotics of the solutions in question. In [20, 22, 23], this method has been developed further and has been adapted for equations of the second order in time governing wave propagation. Global in time solutions of the classical Cauchy problems for (1.2) have been constructed in the form of a series in small parameter present in the initial conditions. The main term of the asymptotics has been presented in the explicit form and has not contained a series in small parameter as in [18]. The questions of the existence and uniqueness of the generalized solutions in the cases of one, two, and three space dimensions have been considered in [21]. However, the asymptotic behavior of solutions to (1.2) has been established only for the case of one space dimension (see [20, 22]). In particular, in [22], it was proved that the major term, as $t \longrightarrow+\infty$, of the solution consisted of two solitary waves traveling in opposite directions and diffusing in space. Each of them was governed by the Burgers equation with a transfer. This long time approximation turned out to be essentially nonlinear. In the present note, it is proved that the linear operator determines the long time behaviour of the solution in the case of two space dimensions (this is usually true also for higher-dimensional cases). The "linear approximation" consists of two parts corresponding to the isotropic and non-isotropic transfer of small perturbations in space. The "non-isotropic" part results from taking into account several terms of the expansions of the Fourier images of the initial data for small values of parameter. 
This, in its own turn, is needed because of the appearance of singularities in the Fourier image representation. Such a situation has never occurred in comparatively simple cases studied in [18]. The integrals present in the major term of the asymptotic formula can be called "modified error functions" and they are interesting from the point of view of the application of Laplace's method in the case of the coalescence of several singularities.

2. Statement of the problem and the main theorem. We pose the following Cauchy problem:

$$
\begin{aligned}
u_{t t}-2 b \Delta u_{t} & =-\alpha \Delta^{2} u+\Delta u+\beta \Delta\left(u^{2}\right), \quad x \in \mathbb{R}^{2}, t>0, \\
u(x, 0) & =\varepsilon \phi(x), \quad u_{t}(x, 0)=\varepsilon \psi(x), \quad x \in \mathbb{R}^{2},
\end{aligned}
$$

where $\alpha, b, \varepsilon=$ const $>0, \beta=$ const $\in \mathbb{R}^{1}, \Delta=\partial^{2} / \partial x_{1}{ }^{2}+\partial^{2} / \partial x_{2}{ }^{2}$, and $\phi(x)$ and $\psi(x)$ are real-valued functions of $x$.

In the sequel, we denote $\int_{\mathbb{R}^{2}}$ by $\iint$, a Fourier transform of the function $u(x, t)$ by

$$
\hat{u}(p, t)=\iint e^{-i(p, x)} u(x, t) d x,(p, x)=p_{1} x_{1}+p_{2} x_{2}, \quad p \in \mathbb{R}^{2},
$$

an inverse transform by

$$
u(x, t)=F^{-1}(\hat{u}(p, t))=(2 \pi)^{-2} \iint e^{i(p, x)} \hat{u}(p, t) d p,
$$

and a convolution in $p$ by

$$
f(p, t) * g(p, t)=\iint f(p-q, t) g(q, t) d q .
$$

We, also, use the space $H^{0}\left(\mathbb{R}^{2}\right)$ equipped with the norm

$$
\|u\|=\|u\|_{H^{0}\left(\mathbb{R}^{2}\right)}=\left(\iint|\hat{u}(p, t)|^{2} d p\right)^{1 / 2}
$$

and the notations $m(p)=\min (1,|p|), M(p)=\max (1,|p|),|p|=\left(p_{1}{ }^{2}+p_{2}{ }^{2}\right)^{1 / 2}$, so that $|p|=m(p) M(p)$. We denote by $c$ different positive constants not dependent on $x, t, \varepsilon$, and $p$. They may depend on the coefficients of the equation and the initial data.

DEFinition. $f(x) \in \widetilde{C}_{x_{1}}{ }^{n}\left(\mathbb{R}^{2}\right)$ if the functions $f(x), \partial f / \partial x_{1}, \ldots, \partial^{n-1} f / \partial x_{1}{ }^{n-1} \longrightarrow$ 0 as $\left|x_{1}\right| \longrightarrow+\infty$, are absolutely integrable over $\mathbb{R}^{1}$ in $x_{2}$, and $\partial^{n} f / \partial x_{1}{ }^{n} \in L_{1}\left(\mathbb{R}^{2}\right)$.

The class $\widetilde{C}_{x_{2}}^{n}\left(\mathbb{R}^{2}\right)$ is defined analogously.

THEOREM 2.1. Let

(A) $\alpha>b^{2}$;

(B) $\underset{\widetilde{C}}{\phi}(x) \in \tilde{C}_{x_{1}}{ }^{7}\left(\mathbb{R}^{2}\right) \cap \widetilde{C}_{x_{2}}{ }^{7}\left(\mathbb{R}^{2}\right), \psi(x) \in \tilde{C}_{x_{1}}{ }^{6}\left(\mathbb{R}^{2}\right) \cap \tilde{C}_{x_{2}}{ }^{6}\left(\mathbb{R}^{2}\right), x(x) \in \tilde{C}_{x_{1}}{ }^{5}\left(\mathbb{R}^{2}\right) \cap$ $\widetilde{C}_{x_{2}}{ }^{5}\left(\mathbb{R}^{2}\right)$.

Then there is such $\varepsilon_{0}>0$ that, for $0<\varepsilon \leq \varepsilon_{0}$, there exists a unique classical solution of the problem (2.1), (2.2) represented as

$$
u(x, t)=\sum_{N=0}^{\infty} \varepsilon^{N+1} u^{(N)}(x, t),
$$


where the functions $u^{(N)}(x, t)$ will be defined in the proof (see (3.8), (3.9), and (3.22)). If, moreover,

(C) $\hat{\phi}(0)=\iint \psi(x) d x=0, \iint\left|x^{2} \psi(x)\right| d x<+\infty, \iint|x \phi(x)| d x<+\infty$, this solution has the following asymptotics as $t \longrightarrow+\infty, x \in \mathbb{R}^{2} \backslash 0$ :

$$
\begin{aligned}
u(x, t)=\varepsilon\{ & \Omega(\theta) \frac{t}{|x|} \frac{1}{\pi \sqrt{\pi b t}}\left[\mathrm{I}_{2}^{-}(\rho, \eta)-\mathrm{I}_{2}{ }^{+}(\omega, \eta)\right] \\
& +\left[\hat{\phi}(0)+\Omega(\theta) \frac{t}{|x|}\right] \frac{1}{2 \pi \sqrt{\pi b t}}\left[\mathrm{I}_{1}^{-}(\rho, \eta)-\mathrm{I}_{1}^{+}(\omega, \eta)\right] \\
& \left.+\Omega(\theta) \frac{1}{|x|} \frac{1}{2 \pi \sqrt{\pi b t}}\left[\mathrm{I}_{0}^{-}(\rho, \eta)-\mathrm{I}_{0}^{+}(\omega, \eta)\right]\right\}+O\left(t^{-3 / 2} \ln t\right),
\end{aligned}
$$

where

$$
\begin{gathered}
\Omega(\theta)=\left(\hat{\Psi}, e_{x}\right), \quad \hat{\Psi}=\left\{\hat{\psi}_{1}, \hat{\psi}_{2}\right\}, \\
\hat{\phi}(0)=\iint \phi(x) d x, \quad \hat{\psi}_{k}=\iint x_{k} \tilde{\psi}(x) d x, \quad k=1,2, \\
e_{x}=x /|x|=\{\cos \theta, \sin \theta\}, \quad \theta \text { is a polar angle on the } x \text {-plane, } \\
\mathrm{I}_{k}^{-}(\rho, \eta)=\int_{0}^{\infty}\left(\eta z^{2}-\rho\right)^{k} \exp \left[-\left(\eta z^{2}-\rho\right)^{2}\right]\left(z^{2}+2\right)^{-1 / 2} d z, \\
\mathrm{I}_{k}{ }^{+}(\omega, \eta)=\int_{0}^{\infty}\left(\eta z^{2}+\omega\right)^{k} \exp \left[-\left(\eta z^{2}+\omega\right)^{2}\right]\left(z^{2}+2\right)^{-1 / 2} d z, \\
k=0,1,2, \quad \rho=\frac{t-|x|}{2 \sqrt{b t}}, \quad \eta=\frac{|x|}{2 \sqrt{b t}}, \quad \omega=\frac{t+|x|}{2 \sqrt{b t}},
\end{gathered}
$$

and the estimate of the residual term is uniform in $x \in \mathbb{R}^{2}$.

REMARK 1. Assumption (A) corresponds to the most interesting case of the existence of damped oscillations. When the relation $0<\alpha<b^{2}$ holds, the solution of the Cauchy problem exhibits basically parabolic traits, and aperiodic processes play the main role. The critical case $\alpha=b^{2}$ needs a special consideration since the linear operator in (1.2) factorizes and becomes $\left(\partial / \partial t-b \partial^{2} / \partial x^{2}-\partial / \partial x\right)\left(\partial / \partial t-b \partial^{2} / \partial x^{2}+\partial / \partial x\right)$ (see $[23,21])$. We do not examine below the last two cases in order not to go into details. All the considerations are absolutely analogous to the ones conducted below.

REMARK 2. Assumption (B) guarantees the fast decay of the Fourier images of the initial data as $|p| \longrightarrow+\infty$, namely, $\hat{\phi}(p)=O\left(|p|^{-7}\right), \hat{\psi}(p)=O\left(|p|^{-6}\right)$, and are needed to secure the necessary smoothness of the constructed solution (see (2.7)).

REMARK 3. We do not calculate the asymptotic form, as $\varepsilon \longrightarrow 0$, of the solution in question. This small parameter is needed for the absolute and uniform convergence of the series in (2.2) and its derivatives included in the equation.

REMARK 4. Assumptions (C) allow us to obtain the necessary asymptotic expansions of the Fourier images of the initial functions as $p \longrightarrow 0$. Together with the expansions of the eigenvalues of the linear operator for small $p$, they are essentially used for calculating long time asymptotics of the solution. 


\section{Proof of the Theorem}

(i) EXISTENCE AND CONSTRUCTION OF THE SOLUTION. Applying the Fourier transform in $x$ to (2.1) and (2.2), we get the following problem for the Fourier image $\hat{u}(p, t)$ :

$$
\begin{gathered}
\hat{u}_{t t}+2 b|p|^{2} \hat{u}_{t}+\left(\alpha|p|^{4}+|p|^{2}\right) \hat{u}=-\beta|p|^{2} \iint \hat{u}(p-q, t) \hat{u}(q, t) d q, t>0, \\
\hat{u}(p, 0)=\varepsilon \hat{\phi}(p), \quad \hat{u}(p, 0)=\varepsilon \hat{\psi}(p) .
\end{gathered}
$$

Choosing the fundamental system of solutions of the homogeneous equation associated with (3.1) in the form $\exp (-\lambda t)$, we obtain the roots of the characteristic equation

$$
\lambda_{1,2}(p)=b|p|^{2} \mp i \sigma(p), \quad \sigma(p)=|p| \sqrt{k|p|^{2}+1},
$$

where $k=\alpha-b^{2}>0$, according to assumption (A) of the hypothesis.

Integrating by parts in $x_{1}$ and $x_{2}$ in the integral representations of $\hat{\phi}(p)$ and $\hat{\psi}(p)$ and taking into account assumption (B), we get, for $p \in \mathbb{R}^{2}$,

$$
|\hat{\phi}(p)| \leq c M^{-7}(p), \quad|\hat{\psi}(p)| \leq c M^{-6}(p) .
$$

Applying Taylor's theorem, we can write that, for $|p| \leq 1, p \in \mathbb{R}^{2}$,

$$
\begin{aligned}
& \hat{\phi}(p)=\hat{\phi}(0)+O(|p|), \\
& \hat{\psi}(p)=-i p_{1} \hat{\psi}_{1}-i p_{2} \hat{\psi}_{2}+O\left(|p|^{2}\right)=-i(\hat{\Psi}, p)+O\left(|p|^{2}\right),
\end{aligned}
$$

where the integrals representing $\hat{\phi}(0), \hat{\psi}_{k}, k=1,2$, and the constants in the residual terms converge absolutely, according to (C). Integrating (3.1) with respect to $t$, we reduce it to the integral equation

$$
\begin{aligned}
\hat{u}(p, t)=\varepsilon \exp \left(-b|p|^{2} t\right)\{ & {\left[\cos (\sigma(p) t)+b|p|^{2}(\sigma(p))^{-1} \sin (\sigma(p) t)\right] \hat{\phi}(p) } \\
& \left.+(\sigma(p))^{-1} \sin (\sigma(p) t) \hat{\psi}(p)\right\} \\
-\beta|p|^{2}(\sigma(p))^{-1} \int_{0}^{t} & \exp \left(-b|p|^{2}(t-\tau)\right) \sin (\sigma(p)(t-\tau)) \hat{u}(p, \tau) * \hat{u}(p, \tau) d \tau .
\end{aligned}
$$

For solving (3.6), we use the perturbation theory. We represent $\hat{u}(p, t)$ in the form of a formal power series in $\varepsilon$

$$
\hat{u}(p, t)=\sum_{N=0}^{\infty} \varepsilon^{N+1} \hat{v}^{(N)}(p, t) .
$$

Inserting (3.7) into (3.6) and equating coefficients of equal powers of $\varepsilon$, we find

$$
\begin{aligned}
\hat{v}^{(0)}(p, t)=\exp \left(-b|p|^{2} t\right)\{[ & \left.\cos (\sigma(p) t)+b|p|^{2}(\sigma(p))^{-1} \sin (\sigma(p) t)\right] \hat{\phi}(p) \\
+ & \left.(\sigma(p))^{-1} \sin (\sigma(p) t) \hat{\psi}(p)\right\}, \\
\hat{v}^{(N)}(p, t)=-\beta|p|^{2}(\sigma(p))^{-1} \int_{0}^{t} & \exp \left(-b|p|^{2}(t-\tau)\right) \sin (\sigma(p)(t-\tau)) \\
& \times \sum_{j=1}^{N} \hat{v}^{(j-1)}(p, \tau) * \hat{v}^{(N-j)}(p, \tau) d \tau, \quad N \geq 1 .
\end{aligned}
$$


Next, we get the following estimates for $t>0, p \in \mathbb{R}^{2}$ :

$$
\begin{aligned}
\left|\hat{v}^{(0)}(p, t)\right| & \leq c \exp [\kappa(p) t] M^{-7}(p), \\
\left|\hat{v}^{(1)}(p, t)\right| & \leq\left. c m(p) \ln |1+| p\right|^{-2} \mid M^{-7}(p) \exp [-\kappa(p / 2) t], \\
\left|\hat{v}^{(N)}(p, t)\right| & \leq c^{N} m(p) M^{-7}(p) \exp [-\kappa(p /(N+1))], \quad N \geq 2,
\end{aligned}
$$

where $\kappa(p)=b|p|^{2} / 2$. They help to justify the formal procedure of constructing the solution and to obtain its long time asymptotics.

We note that, for $p, q \in \mathbb{R}^{2}, N \geq 1,1 \leq j \leq N$, the following inequalities hold (see [18]):

$$
\begin{gathered}
M^{-7}(p-q) M^{-7}(q) \leq 2^{7} M^{-7}(p)\left[M^{-7}(q)+M^{-7}(p-q)\right], \\
j^{-5}(N+1-j)^{-5} \leq 2^{5}(N+1)^{-5}\left[j^{-5}+(N+1-j)^{-5}\right], \\
\kappa((p-q) / j)+\kappa(q /(N+1-j)) \geq \kappa(p /(N+1)) .
\end{gathered}
$$

As regards (3.10), it follows immediately from (3.3)-(3.5), (3.8), and the inequality $b|p|^{2} / \sigma(p) \leq c<\infty$. In order to prove (3.11), we use (3.10), (3.13), and write the following chain of inequalities:

$$
\begin{aligned}
\left|\hat{v}^{(1)}(p, t)\right| & \leq \frac{\beta|p|}{\sqrt{k|p|^{2}+1}} \int_{0}^{t} \exp \left[-b|p|^{2}(t-\tau)\right] \hat{v}^{(0)}(p, \tau) * \hat{v}^{(0)}(p, \tau) d \tau \\
& \leq c m(p) \iint M^{-7}(p-q) M^{-7}(q) S_{1}(p, q, t) d q \\
& \leq c m(p) M^{-7}(p) \iint\left[M^{-7}(p-q)+M^{-7}(q)\right] S_{1}(p, q, t) d q, \\
S_{1}(p, q, t) & =\exp (-2 \kappa(p) t) \int_{0}^{t} \exp [2 \kappa(p) \tau-\kappa(p-q) \tau-\kappa(q) \tau] d \tau .
\end{aligned}
$$

It follows from (3.13) with $N=j=1$ (see [20, 22]) that

$$
S_{1}(p, q, t) \leq c \exp [-\kappa(p / 2) t] /[\kappa(p)+\kappa(q)] .
$$

The convergence of the integral in $q$ on the right-hand side of (3.14) for all $|p| \geq 1$ is evident. To find its singularity for small $p$, it suffices to consider the behavior of the corresponding integral over the domain $|q| \leq 1$. Since $\int_{0}^{1}\left(|p|^{2}+|q|^{2}\right)^{-1}|q| d|q|=$ $1 /\left.2 \ln |1+| p\right|^{-2} \mid$, the inequality (3.11) follows from (3.14) and (3.15).

Now, we establish (3.12) by induction on the number $N$. First, we show that it holds for $N=2$. Using (3.9), we can write

$$
\left|\hat{\nu}^{(2)}(p, t)\right| \leq c m(p) \int_{0}^{t} \exp (-2 \kappa(p)(t-\tau))\left|\hat{\nu}^{(0)}(p, \tau)\right| *\left|\hat{\nu}^{(1)}(p, \tau)\right| d \tau
$$

and estimating the integral

$$
\begin{aligned}
S_{2}(p, q, t) & =\exp (-2 \kappa(p) t) \int_{0}^{t} \exp [2 \kappa(p) \tau-\kappa(p-q) \tau-\kappa(q / 2) \tau] d \tau \\
& \leq \frac{c \exp (-\kappa(p / 3) t)}{\kappa(p)+\kappa(q)}
\end{aligned}
$$


by means of (3.10), (3.11), and (3.13), we get

$$
\begin{aligned}
\left|\hat{v}^{(2)}(p, t)\right| \leq & c m(p) M^{-7}(p) \exp (-\kappa(p / 3) t) \\
& \times \int_{0}^{\infty} \frac{\left.m(q) \ln |1+| q\right|^{-2} \mid}{|p|^{2}+|q|^{2}}\left[M^{-7}(p-q)+M^{-7}(q)\right]|q| d|q| .
\end{aligned}
$$

The last integral converges uniformly in $p \in \mathbb{R}^{2}$. Thus, (3.12) is established for $N=2$.

Assuming that (3.12) is proved for all $\hat{v}^{(s)}(p, t), 2 \leq s \leq N-1$, we show that it holds for $\hat{v}^{(N)}(p, t)$. Indeed,

$$
\begin{aligned}
\left|\hat{v}^{(N)}(p, t)\right| \leq & 2^{12}(N+1)^{-5} M^{-7}(p) m(p) \sum_{j=1}^{N} c^{j-1} c^{N-j}\left[(N+1-j)^{-5}+j^{-5}\right] \\
& \times \iint\left[M^{-7}(p-q)+M^{-7}(q)\right] m(p-q) m(q) S_{N}(p, q, t) d q, \\
S_{N}(p, q, t)= & \exp (-2 \kappa(p) t) \int_{0}^{t} \exp \left[L_{N}(p, q) \tau\right] d \tau, \\
L_{N}(p, q)= & 2 \kappa(p)-\kappa((p-q) / j)-\kappa(q /(N+1-j)) .
\end{aligned}
$$

Denoting a typical term on the right-hand side of the last inequality by $W(p, t)$ and using the estimate (see [18])

$$
S_{N}(p, q, t) \leq \frac{\exp [-\kappa(p /(N+1)) t]}{\max \left[\kappa(p), L_{N}(p, q)\right]} \leq 8(N+1-j)^{2} \frac{\exp [-\kappa(p /(N+1)) t]}{\kappa(p)+\kappa(q)},
$$

we can write

$$
\begin{aligned}
W(p, t) \leq & c^{N-1} M^{-7}(p) \exp [-\kappa(p /(N+1)) t] m(p) \sum_{j=1}^{N}(N+1-j)^{-3} \\
& \times \int_{0}^{\infty}\left[M^{-7}(p-q)+M^{-7}(q)\right] \frac{m(p-q) m(q)}{|p|^{2}+|q|^{2}}|q| d|q| .
\end{aligned}
$$

The estimate (3.12) follows by induction from the uniform convergence in $p \in \mathbb{R}^{2}$ of the last integral. Applying the inverse Fourier transform to (3.7), we find that

$$
\begin{aligned}
u(x, t) & =(2 \pi)^{-2} \iint e^{i(p, x)} \sum_{N=0}^{\infty} \varepsilon^{N+1} \hat{v}^{(N+1)}(p, t) d p \\
& =\sum_{N=0}^{\infty} \varepsilon^{N+1} u^{(N)}(x, t), \\
u^{(N)}(x, t) & =(2 \pi)^{-2} \iint e^{i(p, x)} \hat{v}^{(N)}(p, t) d p .
\end{aligned}
$$

Here, we have performed the termwise integration of the series which is possible due to its absolute and uniform convergence for $\varepsilon<1 / c$. The last statement, in its own turn, follows from the estimates (3.10)-(3.12). By means of these estimates, it can be verified straigtforwardly that (3.22) is indeed a classical solution of the problem (2.1), (2.2) with sufficiently small $\varepsilon$. 
(ii) UNIQUENESS OF THE SOLUTION. Now, we prove the uniqueness of the constructed solution. We assume the contrary, that is, that there exist two classical solutions $u_{1}(x, t)$ and $u_{2}(x, t)$ of the problem in question (note that both of them belong to the space $H^{0}\left(\mathbb{R}^{2}\right)$ for each fixed $\left.t>0\right)$ and set $W(x, t)=u_{1}(x, t)-u_{2}(x, t)$. Applying the Fourier transform in $x$, we get, for $\hat{w}(p, t)$, the following integral equation:

$$
\begin{aligned}
\hat{w}(p, t)=-\left[\beta|p|^{2} / \sigma(p)\right] \int_{0}^{t} & \exp \left[-b|p|^{2}(t-\tau)\right] \sin (\sigma(p)(t-\tau)) \\
& \times\left[\hat{u}_{1}(p, \tau)-\hat{u}_{2}(p, \tau)\right] *\left[\hat{u}_{1}(p, \tau)+\hat{u}_{2}(p, \tau)\right] d \tau .
\end{aligned}
$$

Estimating the right-hand side of this equation by means of Cauchy-Schwartz inequality and using the fact that $|p|^{2} / \sigma(p) \leq c<+\infty$ for all $p \in \mathbb{R}^{2}$ and $\left\|u_{1,2}(t)\right\| \leq c<+\infty$ for all $t>0$, we find that

$$
\begin{aligned}
|\hat{w}(p, t)| & \leq c \int_{0}^{t} \exp \left[-b|p|^{2}(t-\tau)\right]\|W(\tau)\|\left[\left\|u_{1}(\tau)\right\|+\left\|u_{2}(\tau)\right\|\right] d \tau \\
& \leq c \int_{0}^{t} \exp \left[-b|p|^{2}(t-\tau)\right]\|W(\tau)\| d \tau .
\end{aligned}
$$

Squaring both sides of the last inequality and integrating the result in $p$ over $\mathbb{R}^{2}$, we obtain

$$
\|W(t)\|^{2} \leq c \iint\left\{\int_{0}^{t} \exp \left[-b|p|^{2}(t-\tau)\right]\|W(\tau)\| d \tau\right\}^{2} d p
$$

Hence, for some $T_{1}>0$

$$
\begin{aligned}
\left(\sup _{t \in\left[0, T_{1}\right]}\|W(t)\|\right)^{2} & \leq c\left(\sup _{t \in\left[0, T_{1}\right]}\|W(t)\|\right)^{2} \iint\left[\frac{1-\exp \left(-b|p|^{2} t\right)}{b|p|^{2}}\right]^{2} d p \\
& \leq C\left(T_{1}\right)\left(\sup _{t \in\left[0, T_{1}\right]}\|W(t)\|\right)^{2},
\end{aligned}
$$

where the constant $C\left(T_{1}\right)$ can be made less than one by the appropriate choice of $T_{1}$. This contradiction allows us to complete the proof of the uniqueness of the solution for $t \in\left[0, T_{1}\right]$. Continuing this process for the segments $\left[T_{1}, T_{2}\right],\left[T_{2}, T_{3}\right], \ldots$, with $\left\{T_{k}\right\} \longrightarrow+\infty$, we obtain the same result for all $t>0$.

(iii) LONG TIME ASYMPTOTICs. We pass to the calculation of the long time asymptotics of the constructed solution. For this purpose, we first deduce crude long time estimates of $u^{(N)}(x, t)$ with $N \geq 1$ (see (3.22)), which contribute to the remainder of the asymptotic formula and then find a subtle asymptotic estimate of $u^{(0)}(x, t)$ which gives us its major term. Making use of (3.11), we can write

$$
\begin{aligned}
\left|u^{(1)}(x, t)\right| & =(2 \pi)^{-2}\left|\iint e^{i(p, x)} \hat{v}^{(1)}(p, t) d p\right| \\
& \leq\left.\int_{0}^{\infty} \exp \left(-b|p|^{2} t\right) m(p) \ln |1+| p\right|^{-2}\left|M^{-7}(p)\right| p|d| p \mid \\
& =O\left(t^{-3 / 2} \ln t\right) \quad \text { as } t \longrightarrow+\infty .
\end{aligned}
$$


By means of (3.12), we find that

$$
\begin{aligned}
& \left|\sum_{N=2}^{\infty} \varepsilon^{N+1} u^{(N)}(x, t)\right| \\
& \quad \leq \sum_{N=2}^{\infty} c^{N} \varepsilon^{N+1}(N+1)^{-5} \int_{0}^{\infty} \exp \left\{-b|p|^{2} t /\left[2(N+1)^{2}\right]\right\} m(p) M^{-7}(p)|p| d|p| \\
& \quad=O\left(t^{-3 / 2}\right) \sum_{N=2}^{\infty} c^{N} \varepsilon^{N+1}(N+1)^{-2} \\
& \quad=O\left(t^{-3 / 2}\right) \text { as } t \rightarrow+\infty .
\end{aligned}
$$

Note that the constants in the last two estimates depend on $b$. Moreover, $c=c(b) \longrightarrow$ $+\infty$ as $b \longrightarrow 0$. In order to obtain a subtle asymptotic estimate of the term $u^{(0)}(x, t)=$ $F^{-1}\left(\hat{v}^{(0)}(p, t)\right)$, we represent $\hat{v}^{(0)}(p, t)$ as

$$
\begin{aligned}
\hat{v}^{(0)}(p, t) & =\sum_{j=1}^{5} \hat{w}_{j}(p, t), \\
\hat{w}_{1}(p, t) & =\hat{\phi}(0) \exp \left(-b|p|^{2} t\right) \cos (|p| t), \\
\hat{w}_{2}(p, t) & =[\hat{\phi}(p)-\hat{\phi}(0)] \exp \left(-b|p|^{2} t\right) \cos (\sigma(p) t), \\
\hat{w}_{3}(p, t) & =\hat{\phi}(0) \exp \left(-b|p|^{2} t\right)[\cos (\sigma(p) t)-\cos (|p| t)], \\
\hat{w}_{4}(p, t) & =\hat{\phi}(p) \exp \left(-b|p|^{2} t\right) b|p|^{2}[\sigma(p)]^{-1} \sin (\sigma(p) t), \\
\hat{w}_{5}(p, t) & =\hat{\psi}(p) \exp \left(-b|p|^{2} t\right)[\sigma(p)]^{-1} \sin (\sigma(p) t) .
\end{aligned}
$$

For estimating the Fourier transforms of these terms, it suffices to study the integrals only over the domain $|p| \leq 1$ due to the presence of the exponential multipliers. Evidently, the corresponding integrals over $|p| \geq 1$ are $O\left(e^{-b t}\right)$ for all $t>0$. Therefore, we use the asymptotic expansions valid for $|p| \leq 1$. Taking into account (3.4) and the inequalities

$$
|\cos (\sigma(p) t)-\cos (|p| t)| \leq c|p|^{3} t, \quad b|p|^{2} / \sigma(p) \leq c m(p)
$$

and performing the inverse Fourier transforms of $\hat{w}_{j}(p, t)$ with $j=2,3$, we find that

$$
F^{-1}\left(\hat{w}_{j}(p, t)\right)=O\left(t^{-3 / 2}\right), \quad j=2,3,4 \text {, as } t \longrightarrow+\infty \text {. }
$$

Analogously, denoting $\tilde{w}_{5}(p, t)=-i(\hat{\Psi}, p /|p|) \exp \left(-b|p|^{2} t\right) \sin (|p| t)$ with $\hat{\Psi}=\left\{\hat{\psi}_{1}\right.$, $\left.\hat{\psi}_{2}\right\}$ and recalling the asymptotic formula (3.5), we can easily deduce that as $t \rightarrow+\infty$

$$
F^{-1}\left(\hat{w}_{5}\right)=F^{-1}\left(\tilde{w}_{5}\right)+O\left(t^{-3 / 2}\right) .
$$

Thus, it remains to calculate the inverse Fourier transforms of $\hat{w}_{1}(p, t)$ and $\tilde{w}_{5}(p, t)$ in order to find the major term of the long time asymptotics. We need the following 
auxiliary results (see $[10,24])$ :

$$
\begin{gathered}
\int_{0}^{\infty} \exp (i A \cos \gamma) d \gamma=2 \pi J_{0}(A), \\
\int_{0}^{2 \pi} \exp [i(A \cos \gamma+B \sin \gamma)] \cos \gamma d \gamma=i 2 \pi A \frac{J_{1}\left(\sqrt{A^{2}+B^{2}}\right)}{\sqrt{A^{2}+B^{2}}}, \\
\int_{0}^{2 \pi} \exp [i(A \cos \gamma+B \sin \gamma)] \sin \gamma d \gamma=i 2 \pi B \frac{J_{1}\left(\sqrt{A^{2}+B^{2}}\right)}{\sqrt{A^{2}+B^{2}}},
\end{gathered}
$$

where $A, B \in \mathbb{R}^{1}$, and $J_{v}(z), v=0,1$, are Bessel functions of the corresponding index, and the formulas

$$
\begin{gathered}
I(A, B)=\int_{0}^{\infty} \exp \left(-A x^{2}\right) \cos (B x) d x=1 / 2 \sqrt{\pi / A} \exp \left(-B^{2} / 4 A\right), \\
\int_{0}^{\infty} x \exp \left(-A x^{2}\right) \sin (B x) d x=-\partial I(A, B) / \partial B \\
\int_{0}^{\infty} x^{2} \exp \left(-A x^{2}\right) \cos (B x) d x=-\partial^{2} I(A, B) / \partial B^{2}
\end{gathered}
$$

where $A>0, B \in \mathbb{R}^{1}$.

For the fixed $x$, we can direct the horizontal axis of the coordinate system along this vector, so that $(p, x)=|p||x| \cos \varphi$, and write that

$$
\begin{aligned}
F^{-1}\left(\hat{w}_{1}\right) & =(2 \pi)^{-2} \hat{\phi}(0) \int_{0}^{2 \pi} d \varphi \int_{0}^{\infty} \exp \left(i|p||x| \cos \varphi-b|p|^{2} t\right) \cos (|p| t)|p| d|p| \\
& =(2 \pi)^{-1} \hat{\phi}(0) \int_{0}^{\infty} \exp \left(-b|p|^{2} t\right) \cos (|p| t) J_{0}(|p||x|)|p| d|p| .
\end{aligned}
$$

Leaving (3.35) temporarily, we simplify $F^{-1}\left(\hat{w}_{5}\right)$. Introducing the polar coordinates on the $p$-plane, according to the formulas $p_{1}=|p| \cos \vartheta, p_{2}=|p| \sin \vartheta$, and using (3.33), we obtain

$$
\begin{aligned}
F^{-1}\left(\tilde{w}_{5}\right)= & (2 \pi)^{-2} \iint e^{i(p, x)} \tilde{w}_{5}(p, t) d p \\
= & -i(2 \pi)^{-2} \iint(\hat{\Psi}, p /|p|) \exp \left[i(p, x)-b|p|^{2} t\right] \sin (|p| t) d p \\
= & -(2 \pi)^{-2} \int_{0}^{\infty} \exp \left(-b|p|^{2} t\right) \sin (|p| t)|p| d|p| \\
& \times \int_{0}^{2 \pi} \exp \left[i|p|\left(x_{1} \cos \vartheta+x_{2} \sin \vartheta\right)\right]\left(\hat{\psi}_{1} \cos \vartheta+\hat{\psi}_{2} \sin \vartheta\right) d \vartheta \\
= & \left(\hat{\Psi}, e_{x}\right)(2 \pi)^{-1} \int_{0}^{\infty} \exp \left(-b t r^{2}\right) \sin (t r) J_{1}(r|x|) r d r,
\end{aligned}
$$

where $e_{x}=x /|x|$.

In order to simplify the integrals (3.35), (3.36), we use the Meler-Sonine's representation [10, 24]

$$
J_{0}(A)=\frac{2}{\pi} \int_{1}^{\infty} \frac{\sin (A \xi)}{\sqrt{\xi^{2}-1}} d \xi, \quad A>0
$$


change the order of integration, and calculate the inner integral. Note that direct use of the formula $J_{1}(A)=(2 A / \pi) \int_{0}^{1} \sqrt{1-\xi^{2}} \cos (A \xi) d \xi$ does not permit us to perform one integration. Therefore, we first integrate by parts in (3.36) reducing the index of the Bessel function and then use (3.37).

Taking into account that $J_{1}(r|x|)=(-1 /|x|) \partial J_{0}(r|x|) / \partial r$ and integrating by parts with respect to $r$ in (3.36), we get

$$
\begin{aligned}
& F^{-1}\left(\tilde{w}_{5}\right)=\left(\hat{\Psi}, e_{x}\right)(2 \pi|x|)^{-1}\left[H_{1}(x, t)+H_{2}(x, t)+H_{3}(x, t)\right], \\
& H_{1}(x, t)=(-2 b t) \int_{0}^{\infty} \exp \left(-b t r^{2}\right) \sin (t r) J_{0}(r|x|) r^{2} d r \\
& H_{2}(x, t)=t \int_{0}^{\infty} \exp \left(-b t r^{2}\right) \cos (t r) J_{0}(r|x|) r d r \\
& H_{3}(x, t)=\int_{0}^{\infty} \exp \left(-b t r^{2}\right) \sin (t r) J_{0}(r|x|) d r
\end{aligned}
$$

Combining $H_{2}(x, t)$ with (3.35), using (3.37), changing the order of integration, and calculating the inner integral with the help of (3.34b), we get

$$
\begin{array}{rl}
\mathrm{Y}(x, t) \frac{2}{\pi} \int_{0}^{\infty} \exp \left(-b t r^{2}\right) \cos (t r) r & d r \int_{1}^{\infty} \frac{\sin (r|x| \xi)}{\sqrt{\xi^{2}-1}} d \xi \\
=\mathrm{Y}(x, t) \frac{1}{\sqrt{\pi b t}} \int_{1}^{\infty}\left[\frac{|x| \xi-t}{4 b t} \exp \left(-\frac{(t-|x| \xi)^{2}}{4 b t}\right)\right. \\
\left.+\frac{|x| \xi+t}{4 b t} \exp \left(-\frac{(t+|x| \xi)^{2}}{4 b t}\right)\right] \frac{d \xi}{\sqrt{\xi^{2}-1}}
\end{array}
$$

where $\mathrm{Y}(x, t)=\hat{\phi}(0)+\left(\hat{\Psi}, e_{x}\right) t /|x|$. Since the integral (3.37) converges conditionally for $A>0$ the interchange of the integration performed above must be justified. We can do it by representing the integral in $\xi$ as $\int_{1}^{\infty}=\int_{1}^{R}+\int_{R}^{\infty}$ with some $R>0$ and integrating by parts in $\int_{R}^{\infty}$ improving thus the convergence. Then the usual interchange of integration can be done.

Setting $\sqrt{\xi^{2}-1}=z$ and using the notations of (2.8), we obtain the second term in the braces in (2.7). The integrals $H_{1}(x, t)$ and $H_{3}(x, t)$ can be transformed in an analogous way with the help of (3.34a) and (3.34c). They yield the first and the third terms in the braces. Combining these results with (3.27) and (3.28), we complete the proof of the theorem.

4. Discussion and conclusions. The major term of the long time asymptotics (2.8) looks sufficiently easier than its predecessors, the integrals containing the products of exponents, the Bessel and trigonometric functions, but some efforts are still needed in order to obtain information from it. We begin our analysis with some preliminary remarks. The term containing $\hat{\phi}(0)\left[\mathrm{I}_{1}{ }^{-}(\rho, \eta)-\mathrm{I}_{1}{ }^{+}(\omega, \eta)\right]$ depends only on $(|x|, t)$ and, thus, accounts for the uniform transfer of small perturbations in space. It is shown later that the major changes occur in the vicinity of the surface $\{|x|=t\}$ (which can be called the wave front) and, therefore, these perturbations propagate with a unit speed. The integrals $\mathrm{I}_{k}{ }^{+}(\omega, t), k=0,1,2$, can be called "parasitic terms" and can 
be included in the remainder of the asymptotic formula under some additional assumptions. All the terms in (2.8) containing the function $\Omega(\theta)$ correspond to a nonisotropic transfer of small perturbations. Indeed, $\Omega(\theta)$ can be called a "directional derivative" since $\Omega(\theta)=\hat{\psi}_{1} \cos \theta+\hat{\psi}_{2} \sin \theta, \hat{\psi}_{k}=\partial \hat{\psi}(0,0) / \partial p_{k}, k=1$, 2. This expression may be rewritten as $\Omega(\theta)=|\hat{\Psi}| \sin \left(\theta+\theta_{0}\right), \theta_{0}=\arcsin \left(\hat{\psi}_{1} /|\hat{\Psi}|\right)$. Hence, $\Omega(\theta)=0$ for $\theta_{1}=-\theta_{0}, \theta_{2}=\pi-\theta_{0}$. Thus, for these two angles, only the "isotropic term" remains in (2.8).

In order to simplify (2.8), we consider a special type of asymptotics, when $t \rightarrow+\infty$ on the conical surfaces $|x| / t=v=$ const $>0$. Then

$$
\mathrm{I}_{k}{ }^{+}(\omega, \eta)=O\left(e^{-t / 4 b}\right) \text { as } t \longrightarrow+\infty, k=0,1,2,
$$

and must be included in the residual term of the asymptotics. We denote

$$
\begin{aligned}
Q_{1} & =\{(x, t): 0<|x| / t \leq 1-\delta\}, \\
Q & =\{(x, t):|x| / t=1\}, \\
Q_{2} & =\{(x, t):|x| / t \geq 1+\delta\},
\end{aligned}
$$

where $\delta>0$ is sufficiently small.

Since $\rho=0$ on $Q$, the asymptotic expansions of $\left.\mathrm{I}_{k}{ }^{-}\right|_{Q}, k=0,1,2$, can be calculated by means of Watson's lemma [24]. As a result, we obtain

$$
\left.u\right|_{Q}=\varepsilon \Omega(\theta) C_{1}(b) t^{-3 / 4}+O\left(t^{-5 / 4}\right), \quad t \rightarrow+\infty,
$$

where $C_{1}(b)=\Gamma(1 / 4) \pi^{-3 / 2} b^{-1 / 4}$ and $\Gamma(z)=\int_{0}^{\infty} \xi^{z-1} e^{-\xi} d \xi$ is the usual $\Gamma$-function. Here, we have used the estimates

$$
\begin{aligned}
\left.t^{-1 / 2} \mathrm{I}_{2}{ }^{-}\right|_{Q} & =O\left(t^{-3 / 4}\right), \\
\left.t^{-1} \mathrm{I}_{1}{ }^{-}\right|_{Q} & =O\left(t^{-5 / 4}\right), \\
\left.|x|^{-1} t^{-1 / 2} \mathrm{I}_{0}{ }^{-}\right|_{Q} & =O\left(t^{-7 / 4}\right)
\end{aligned}
$$

and have calculated the constant in the major term of $\mathrm{I}_{2}{ }^{-}$.

In the domain $Q_{1}$, we can rewrite the integrals in question as

$$
\begin{aligned}
\mathrm{I}_{k}{ }^{-} & =t^{k / 2} v^{k} \int_{0}^{\infty}\left(z^{2}-a\right)^{k}\left(z^{2}+2\right)^{-1 / 2} \exp (-\chi S(z, a)), \\
\chi & =\eta^{2}=v^{2} t / 4 b \longrightarrow+\infty, k=0,1,2 ; \\
S(z, a) & =\left(z^{2}-a\right)^{2}, a=\rho / \eta=(1-v) / v \geq \delta /(1-\delta)>0,
\end{aligned}
$$

and consider them from the point of view of applying Laplace's method [11]. Finding a uniform asymptotics of such integrals is difficult because, when $x \rightarrow+\infty$ and $a \longrightarrow 0$, the saddle points (the roots of the equation $\left.S_{z}(z, a)\right) z_{1}=0, z_{2,3}= \pm \sqrt{a}$ coalesce with the end of the integration contour $z_{4}=0$. But we can use Laplace's method in the domain $Q_{1}$ and find that

$$
t^{-1 / 2} \mathrm{I}_{2}{ }^{-}=O\left(t^{-1}\right), \quad t^{-1} \mathrm{I}_{1}{ }^{-}=O\left(t^{-3 / 2}\right), \quad|x|^{-1} t^{-1 / 2} \mathrm{I}_{0}{ }^{-}=O\left(t^{-2}\right), \quad t \rightarrow+\infty .
$$


Therefore, as $v=$ const, $t \longrightarrow+\infty$

$$
u=\varepsilon \Omega(\theta) C_{2}(b, v) t^{-1}+O\left(t^{-3 / 2} \ln t\right)
$$

(we have omitted a rather lengthy expression for $C_{2}(b, v)$ ). However, for the angles $\theta_{1}=-\theta_{0}, \theta_{2}=\pi-\theta_{0}$, the asymptotic formula (2.8) gives only a crude estimate of the solution, namely, $u=O\left(t^{-3 / 2} \ln t\right)$.

In the domain $Q_{2}$, we have $|a|=|\rho| / \eta=(v-1) / v \geq \delta /(1+\delta)>0$ and

$$
\mathrm{I}_{k}^{-}=t^{k / 2} v^{k} e^{-\chi|a|^{2}} \int_{0}^{\infty}\left(z^{2}+|a|\right)^{k}\left(z^{2}+2\right)^{-1 / 2} \exp \left[-\left(z^{4}+2|a| z^{2}\right)\right] d z,
$$

with the same $\chi \longrightarrow+\infty$ as before. As a result, as $v=$ const, $t \rightarrow+\infty$

$$
\begin{aligned}
t^{-1 / 2} \mathrm{I}_{2}{ }^{-} & =\exp \left(-\chi|a|^{2}\right) O(1), \\
t^{-1} \mathrm{I}_{1}{ }^{-} & =\exp \left(-\chi|a|^{2}\right) O\left(t^{-1}\right), \\
|\chi| t^{-1 / 2} \mathrm{I}_{0}{ }^{-} & =\exp \left(-\chi|a|^{2}\right) O\left(t^{-7 / 4}\right)
\end{aligned}
$$

and

$$
u=\varepsilon C_{3}(b, v) e^{-\rho^{2}}\left[\Omega(\theta)+O\left(t^{-1}\right)\right]+O\left(t^{-3 / 2} \ln t\right) .
$$

Although the estimate of the remainder in (2.8) is uniform in space, the presence of the exponential multipliers $\exp \left(-\chi|a|^{2}\right)=\exp \left(-\rho^{2}\right)=\exp \left[-(|x|-t)^{2} / 4 b t\right]$ in the integrals $\mathrm{I}_{k}{ }^{-}$shows that the long-time approximation (2.8) is effective in the zone that can be roughly estimated as ||$x|-t| \leq c \sqrt{t} \ln t$. Outside it, (2.8) gives only a rough estimate of the solution $u=O\left(t^{-3 / 2} \ln t\right)$ as $t \rightarrow+\infty$.

In order to trace the continuous dependence of the integrals $\mathrm{I}_{k}{ }^{-}(\rho, \eta)$ on the parameter $\rho$, we set $\eta z^{2}-\rho=\zeta$ for $\rho \geq 0$ and find that

$$
\begin{aligned}
& \mathrm{I}_{k}{ }^{-}(\rho, \eta)=1 / 2\left[F_{k}(\rho, \eta)+(-1)^{k} g_{k}(\rho, \eta)\right], \\
& F_{k}(\rho, \eta)=\int_{0}^{\infty} \zeta^{k} \exp \left(-\zeta^{2}\right)[(\zeta+\rho)(\zeta+\rho+\eta)]^{-1 / 2} d \zeta \geq 0, \\
& g_{k}(\rho, \eta)=\int_{0}^{\rho} \zeta^{k} \exp \left(-\zeta^{2}\right)[(\rho-\zeta)(\rho-\zeta+2 \eta)]^{-1 / 2} d \zeta, \\
& 0 \leq g_{k}(\rho, \eta) \leq \rho^{k} \ln \left|1+\rho / \eta+\left[(\rho / \eta)^{2}+2 \rho / \eta\right]^{1 / 2}\right|, \quad k=0,1,2 ; \eta>0
\end{aligned}
$$

(we obtained the last bound with the help of the inequality $\zeta^{k} \exp \left(-\zeta^{2}\right) \leq \rho^{k}$ for $0 \leq \zeta \leq \rho)$. Hence, it follows that $\mathrm{I}_{1}{ }^{-}(\rho, \eta)=1 / 2\left[F_{1}(\rho, \eta)-g_{1}(\rho, \eta)\right]$, where $F_{1}(\rho, \eta)$ increases and $g_{1}(\rho, \eta)$ decreases monotonically as $\rho \longrightarrow+0$. Consequently, $\mathrm{I}_{1}{ }^{-}(\rho, \eta)$ reaches its maximum when $\rho=0$ (that is when $|x|=t$ ) and begins to decrease monotonically after passing this point. The second part of this statement follows directly from (2.8). It confirms the remark about the speed of the propagation of uniform in space perturbations made at the beginning of this section.

Note that the restriction $x \neq(0,0)$ (see (2.8)) appears thanks to our use of the MelerSonine's representation of the Bessel functions of the zero index (see (3.37)). It is the price to pay for our method of the simplification of the inverse Fourier transform. 
In conclusion, we would like to point out that the nonlinearity does not contribute to the major part of the asymptotics and, therefore, (2.8) must be considered as a "linear approximation". Passing to the limit, $b \longrightarrow+0$ is impossible since the constant in the estimate of the residual term tends to infinity as $b$ tends to zero. But the dependence on $\varepsilon$ and $\beta$ is continuous, and the constant in the estimate of the remainder is proportionate to $\beta$.

\section{REFERENCES}

[1] M. J. Ablowitz and H. Segur, Solitons and the inverse scattering transform, SIAM Studies in Applied Mathematics, vol. 4, Society for Industrial and Applied Mathematics (SIAM), Philadelphia, 1981. MR 84a:35251. Zbl 472.35002.

[2] M. Abramowitz and I. A. Stegun, Handbook of mathematical functions, with formulas, graphs, and mathematical tables, Edited by Milton Abramowitz and Irene A. Stegun. Third printing, with corrections. National Bureau of Standards Applied Mathematics Series, vol. 55, Superintendent of Documents, U.S. Government Printing Office, Washington, 1965, Handbook: Mathematical functions. MR 31\#1400. Zbl 543.33001.

[3] P. Biler, Regular decay of solutions of strongly damped nonlinear hyperbolic equations, Appl. Anal. 32 (1989), no. 3-4, 277-285. MR 90j:34090. Zbl 666.34072.

[4] _ A singular perturbation problem for nonlinear damped hyperbolic equations, Proc. Roy. Soc. Edinburgh Sect. A 111 (1989), no. 1-2, 21-31. MR 90c:35017. Zbl 701.35013.

[5] _ Time decay of solutions of semilinear strongly damped generalized wave equations, Math. Methods Appl. Sci. 14 (1991), no. 6, 427-443. MR 93c:35011. Zbl 753.35011.

[6] J. Boussinesq, Theorie des ondes et des remous qui se propagent le long d'un canal rectangulaire horizontal, et communiquant au liquide contene dans ce canal des vitesses sensiblement pareilles de la surface au fond, J. Math. Pures Appl. 17 (1872), no. 2, 55-108.

[7] F. Calogero and A. Degasperis, Spectral transform and solitons. Vol. I, vol. 13, Studies in Mathematics and its Applications, no. 144, North-Holland Publishing Co., Amsterdam, 1982, Tools to solve and investigate nonlinear evolution equations. Lecture Notes in Computer Science. MR 84f:35121. Zbl 501.35072.

[8] P. A. Clarkson, New exact solutions of the Boussinesq equation, European J. Appl. Math. 1 (1990), no. 3, 279-300. MR 92d:35244. Zbl 721.35074.

[9] S. Yu. Dobrokhotov, Nonlocal analogues of the nonlinear Boussinesq equation for surface waves over an uneven bottom and their asymptotic solutions, Dokl. Akad. Nauk SSSR 292 (1987), no. 1, 63-67 (Russian). MR 88a:35202. Zbl 626.76023.

[10] A. Erdelyi, W. Magnus, F. Oberhettinger, and F. G. Tricomi, Higher transcendental functions, vol. 1,2, McGraw-Hill Book Company, Inc., London, 1953, Based, in part, on notes left by Harry Bateman. MR 15,419i. Zbl 052.29502.

[11] M. V. Fedoryuk, Asimptotika: integraly i ryady. [Asymptotics: integrals and series], Spravochnaya Matematicheskaya Biblioteka. [Mathematical Reference Library], "Nauka”, Moscow, 1987. MR 89j:41045. Zbl 641.41001.

[12] R. Hirota, Exact $N$-soliton solutions of the wave equation of long waves in shallow-water and in nonlinear lattices, J. Mathematical Phys. 14 (1973), 810-814. MR 493352. Zbl 261.76008 .

[13] Solutions of the classical Boussinesq equation and the spherical Boussinesq equation:the Wronskian technique, J. Phys. Soc. Japan 55 (1986), 2173-2150.

[14] H. A. Levine, Instability and nonexistence of global solutions to nonlinear wave equations of the form $P u_{t t}=-A u+\mathscr{F}(u)$, Trans. Amer. Math. Soc. 192 (1974), 1-21. MR 49 9436. Zbl 288.35003. 
[15] H. A. Levine and B. D. Sleeman, A note on the nonexistence of global solutions of initial-boundary value problems for the Boussinesq equation $u_{t t}=3 u_{x x x x}+$ $u_{x x}-12\left(u^{2}\right)_{x x}$, J. Math. Anal. Appl. 107 (1985), no. 1, 206-210. MR 86i:35127. Zbl 591.35010.

[16] J. W. Miles, Solitary waves, Annual review of fluid mechanics (Calif.), vol. 12, Annual Reviews, 1980, pp. 11-43. MR 82e:76018. Zbl 463.76026.

[17] A. Nakamura, Exact solitary wave solutions of the spherical Boussinesq equation, J. Phys. Soc. Japan 54 (1985), no. 11, 4111-4114. MR 87b:76026.

[18] P. I. Naumkin and I. A. Shishmarev, Nonlinear nonlocal equations in the theory of waves, Translations of Mathematical Monographs, vol. 133, American Mathematical Society, Providence, RI, 1994, Translated from the Russian manuscript by Boris Gommerstadt. MR 94m:35230. Zbl 802.35002.

[19] B. Straughan, Global nonexistence of solutions to some Boussinesq type equations, J. Math. Phys. Sci. 26 (1992), no. 2, 155-164. MR 94a:35100. Zbl 786.35107.

[20] V. V. Varlamov, The asymptotic behavior for large time values of the solution of the Boussinesq equation with dissipation, Comput. Math. Math. Phys. 34 (1994), no. 8-9, 10331043, English translation of the Russian journal. MR 95h:35033. Zbl 835.35116.

[21] _ Existence and uniqueness of a solution to the Cauchy problem for the damped Boussinesq equation, Math. Methods Appl. Sci. 19 (1996), no. 8, 639-649. MR 97e:35167. Zbl 847.35111.

[22] _ On the Cauchy problem for the damped Boussinesq equation, Differential Integral Equations 9 (1996), no. 3, 619-634. MR 97b:35159. Zbl 844.35095.

[23] _ On spatially periodic solutions of the damped Boussinesq equation, Differential Integral Equations 10 (1997), no. 6, 1197-1211.

[24] G. N. Watson, A Treatise on the Theory of Bessel Functions, second ed., The Macmillan Company, New York, 1944. MR 6,64a. Zbl 063.08184.

[25] G. B. Whitham, Comments on periodic waves and solitons, IMA J. Appl. Math. 32 (1984), no. 1-3, 353-366. MR 86d:76008. Zbl 565.35090.

[26] N. Yajima, On a growing mode of the Boussinesq equation, Progr. Theoret. Phys. 69 (1983), no. 2, 678-680. MR 84g:76018.

[27] Y. C. You, Inertial manifolds and stabilization in nonlinear elastic systems with structural damping, Differential equations with applications to mathematical physics (Boston), Academic Press, 1993, pp. 335-346. MR 93k:35260. Zbl 794.93058.

[28] V. E. Zakharov, On stochastization of one-dimentional chains of nonlinear oscillations, Soviet Phys. JETP 38 (1974), 108-110.

VARlamov: Departamento de Matematicas, Escuela Colombiana De Ingenieria A. A. 14520, SANTAFE DE BOGOTA, COLOMBIA 


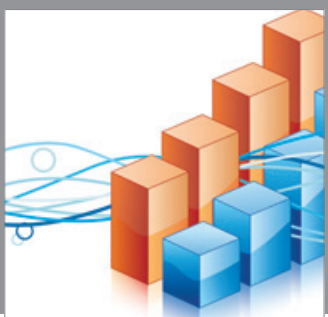

Advances in

Operations Research

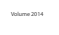

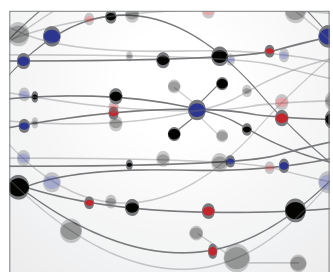

\section{The Scientific} World Journal
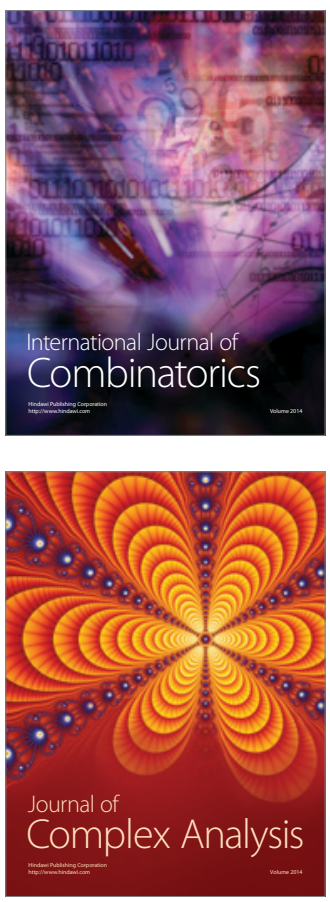

International Journal of

Mathematics and

Mathematical

Sciences
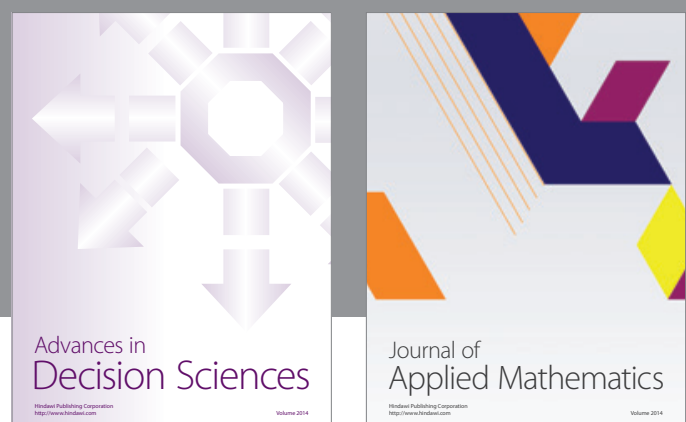

Journal of

Applied Mathematics
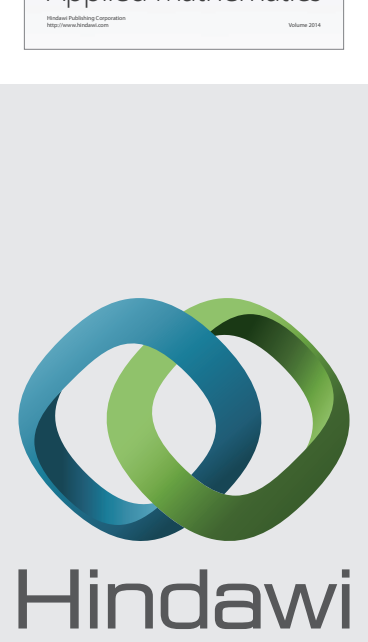

Submit your manuscripts at http://www.hindawi.com
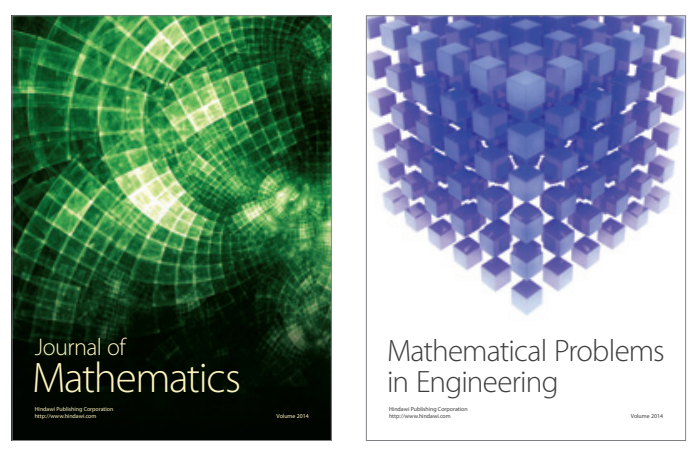

Mathematical Problems in Engineering
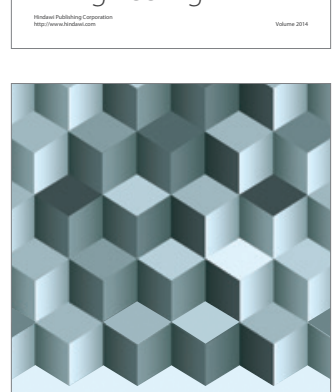

Journal of

Function Spaces
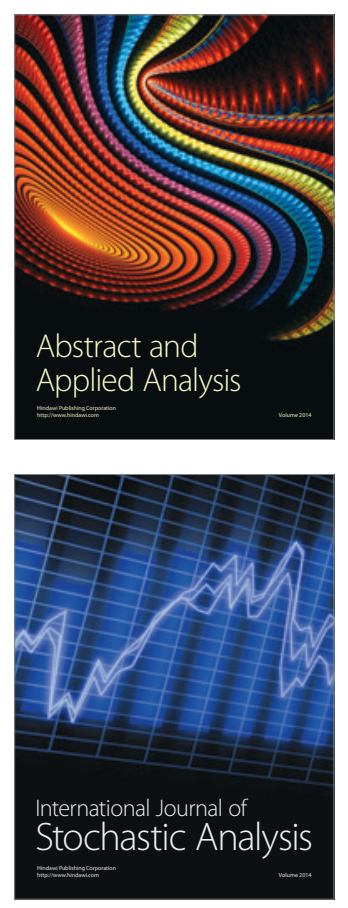

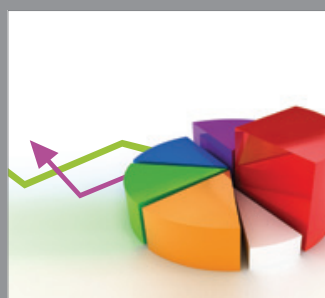

ournal of

Probability and Statistics

Promensencen
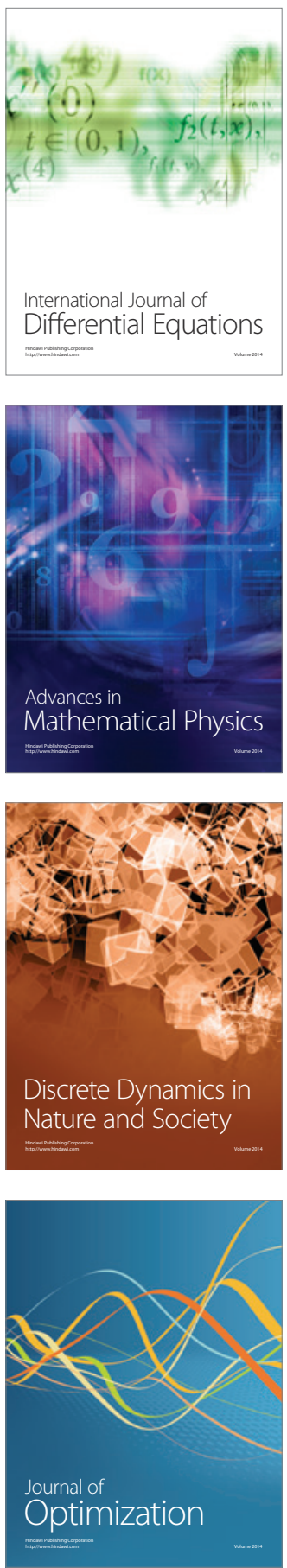